\title{
Betting on Metformin and their Signature Modality in Realm of Cancer Therapy
}

\author{
Karki K, Harrelson S, Branch S and Khan S* \\ Vikor Scientific Biotechnology/KOR Life Sciences, Department of Drug Discovery, USA \\ *Corresponding author: Saeed Khan, Vikor Scientific Biotechnology/KOR Life Sciences, Department of Drug Discovery, and \\ Development, 22 West Edge, USA. \\ To Cite This Article: Karki K, Harrelson S, Branch S, Khan S. Betting on Metformin and their Signature Modality in Realm of Cancer Therapy. \\ Am J Biomed Sci \& Res. 2021 - 13(2). AJBSR.MS.ID.001856. DOI: 10.34297/AJBSR.2021.13.001856.
}

Received: 制 May 27, 2021; Published: 制 June 18, 2021

\section{Introduction}

Natural products and their synthetic analogs have played an integral role in drug discovery, mostly for cancer and other infectious diseases and over $60 \%$ of currently available anticancer drugs are from natural sources [1,2]. Plant-based compounds that show antitumor properties belong to various groups of compounds, such as alkaloids, diterpenes, diterpenoquinone, purine-based compounds, lactonic sesquiterpene, peptides, cyclic depsipeptide, proteins, and macrocyclic polyethers. One of the most famous plant-based drugs is metformin, used as antihyperglycemic drug, which was isolated from Galega officinalis [3,4].

Metformin has been receiving wide attention as a potential anticancer treatment following retrospective results in improving the overall survival rate in cancer patients with diabetes using metformin as a primary medication than non-metformin treatment [5]. The major effects of metformin as an anticancer agent are due to the inactivation of oxidative phosphorylation in mitochondria and activation of AMPK [6,7]. Activation of AMPK directly inhibits mTOR pathway via phosphorylation of TSC1/2, a tumor suppressor gene that negatively regulates mTOR $[7,8]$. Metformin-mediated AMPK activation increases the expression of p53, a tumor suppressor gene that promotes apoptosis and autophagy by inactivating Akt and mTOR protein. Metformin-mediated MAPK activation increases the expression of p53, a tumor suppressor gene that promotes apoptosis and autophagy by inactivating Akt and mTOR protein [9]. Furthermore, metformin inhibits receptor tyrosine kinases EGFR, ErbB2, and IRS1; IRS1 activates IGF1R and PI3/Akt pathway [10-12]. Metformin also inhibits the growth of cancer stem cells and tumor-initiating cells by targeting the sphere-forming ability of CD44+CD24-, CD61highCD49fhigh, CD133+, ALDH1+,
EpCAM+, CD133+CD44+, and CD44+ CD117+ subpopulations in breast, pancreatic, glioblastoma, CRC, and ovarian cancer models. Metformin has also been reported to target various microRNAs (miRNAs), proteins associated with the miRNA biogenesis pathway. As such, metformin inhibits the proliferative capability of breast cancer cells by downregulating miR-27a [13] and upregulating miR-193 (miR-193a-3p and miR193b) [14], which in turn increased AMPK $\alpha$ and decreased FASN levels, respectively. Specificity protein transcription factors (Sp1, Sp3, and Sp4) are non-oncogene addict genes that are highly expressed in solid tumor-derived cells, including pancreatic cancer cells that regulate cancer cell growth, survival, migration/invasion, and inhibit apoptosis [15,16]. Several anticancer agents, including metformin, inhibit colon cancer growth in both in-vitro and in vivo models by downregulating Sp1, Sp3, and Sp4 and Sp-regulated pro-oncogenes such as mTOR and subsequent mTOR downstream genes [17]. This highlights the important chemotherapeutic aspect of metformin, suggesting a huge potential as an anticancer agent against several cancers.

Studies in a combination of metformin with several FDAapproved chemotherapeutics such as gefitinib and cisplatin have demonstrated a significant reduction of tumor burden in mice with lung cancer xenograft models [18]. Few studies performed in pancreatic cancer cells reported that metformin increased the sensitivity of pancreatic cancer cells to gemcitabine treatment by decreasing CD133+ cell populations and inhibiting phosphorylation of ERK/P70S6K signaling and reversing EMT through regulation of miR-663 [19,20]. Several clinical trials are underway to evaluate the therapeutic efficacy of metformin as a single or in combination with FDA-approved drugs in breast, pancreatic, endometrial, lung, colon, and prostate cancers and drugs in combination such as 
cyclophosphamide, doxorubicin, docetaxel, epirubicin, everolimus, exemestane, trastuzumab, atorvastatin, letrozole, megestrol acetate, carboplatin, and fluorouracil (5-FU). The therapeutic efficacy of these drugs combined with metformin is not remarkable because of either minimal to no effect on tumor burden or concern with systemic toxicities [21].

AcompletedPhaseIItrialofmetforminandmedroxyprogesterone acetate combination treatment in endometrial cancer showed modest outcomes with no severe toxicities [22]. Several concerns are against these trials and needed attention. To understand the potential anticancer effects of metformin in clinical settings, the efficacy of metformin as a chemotherapeutic agent still needs investigation.

Despite the potential anticancer effects, the bioavailability of metformin and/or its uptake by cancer cells is too low to exert its antitumor effects in humans. The anticancer effect of metformin in invitro usually falls within the range of 5 to $10 \mathrm{mM}$, which is much higher than steady-state range levels in the plasma of patients with $\mathrm{T} 2 \mathrm{DM}$, which is around $10 \mu \mathrm{M}$ and can reach $40 \mu \mathrm{M}$ when standard prescribed dose is given [23]. Invitro studies show that this low dose is not sufficient to trigger AMPKa activation [24]. Another study highlights the unfavorable pharmacokinetic profile of metformin, suggesting slow and incomplete absorption from the gastrointestinal tract [25].

The pharmacokinetics properties of metformin are determined by its hydrophilic character, and the positive charge under physiological conditions made it difficult for passive diffusion in cells [26]. Organic cation transporters (OCTs) 1, 2, and 3 shuttle metformin inside the cell, and the expression of these transporters are in varying levels in different organs, including the liver, muscle, ovary, and kidney $[27,28]$. ОСТ2 is highly overexpressed in the kidney and is primarily responsible for eliminating metformin via urine [29]. Patients with compromised kidney function seemed to have a short half-life of metformin followed by a wide range of peak to trough drug levels $[27,29]$. Several studies have demonstrated the decreased nephrotoxicity when ОСТ2 inhibitor cimetidine is treated in patients with cisplatin by restricting the effect of cisplatin in the kidney [30-32]. Another study highlights the interindividual genetic variability of OCT1 leading to gastrointestinal side effects [33].

Efforts to improve and enhance the potency of metformin involved modification of structure by attaching alkyl or aromatic groups, but very few showed improved anticancer potency and pharmacokinetics [34]. Another biguanide, phenformin shows 50fold higher anticancer potency than metformin, however, it was removed from the market due to the high risk of fatal lactic acidosis [35]. Drugs inhibiting the OCTs transporter could eventually increase the plasma concentration of metformin, however, it could also lead to metformin-induced lactic acidosis (MALA) [36]. Sulfonamide and sulfenamide derivatives of metformin showed increased potency than the parent molecule in breast and colon cancer models. Similarly, another derivative, HL156A, and triphenylphosphonium cation showed a potential effect in oral squamous cell carcinoma and pancreatic cancer cells via a ROS induction mediated activation of AMPK $\alpha$ and subsequent inhibition of insulin-like growth factor/AKT/mTOR pathway [37,38]. It seems that the antitumor activity of metformin requires higher dose, which exceeds the normal dose required for diabetes patients and that could lead to systemic toxicity.

The collective evidence on the anticancer effects of metformin and its lack of therapeutic concentration in the plasma is summarized here to raise the enthusiasm for further investigation. Several important issues need to be addressed before designing pre-clinical and clinical studies. Metformin analog, NT1014, a novel orally bioavailable drug with increased AMPK activity and increased affinity towards OCT1 and OCT3 showed anticancer activity in in vivo ovarian cancer model [39]. Another analog, HL156A is in a preclinical stage for treating solid tumors [40]. This information suggests that functional derivatives of metformin alone or in combination with other FDA-approved drugs have a huge potential to move forward as an anticancer drug.

\section{References}

1. Kinghorn AD, Chin YW, Swanson SM (2009) Discovery of natural product anticancer agents from biodiverse organisms. Curr Opin Drug Discov Devel 12(2): 189-196.

2. Cragg GM, Newman DJ (2005) Plants as a source of anti-cancer agents. J Ethnopharmacol 100(1-2): 72-79.

3. Lichota A, Gwozdzinski K (2018) Anticancer Activity of Natural Compounds from Plant and Marine Environment. Int J Mol Sci 19(11): 3533.

4. Witters LA (2001) The blooming of the French lilac. J Clin Invest 108(8): 1105-1107.

5. Chae YK, Arya A, Malecek MK, Shin DS, Carneiro B, et al. (2016) Repurposing metformin for cancer treatment: current clinical studies. Oncotarget 7(26): 40767-40780.

6. Morales DR, Morris AD (2015) Metformin in cancer treatment and prevention. Annu Rev Med 66: 17-29.

7. Pernicova I, Korbonits M (2014) Metformin--mode of action and clinical implications for diabetes and cancer. Nat Rev Endocrinol. 10(3): 143-56.

8. Yu X, Mao W, Zhai Y, Tong C, Liu M, et al. (2017) Anti-tumor activity of metformin: from metabolic and epigenetic perspectives. Oncotarget 8(3): 5619-5628.

9. Zhang HH, Guo XL (2016) Combinational strategies of metformin and chemotherapy in cancers. Cancer Chemother Pharmacol 78(1): 13-26.

10. Pierotti MA, Berrino F, Gariboldi M, Melani C, Mogavero A, et al. (2013) Targeting metabolism for cancer treatment and prevention: metformin, an old drug with multi-faceted effects. Oncogene 32(12): 1475-1487.

11. Lei Y, Yi Y, Liu Y, Liu X, Keller ET, et al. (2017) Metformin targets multiple signaling pathways in cancer. Chin J Cancer 36(1): 17. 
12. Zhu P, Davis M, Blackwelder AJ, Bachman N, Liu B, et al. (2014) Metformin selectively targets tumor-initiating cells in ErbB2-overexpressing breast cancer models. Cancer Prev Res (Phila) 7(2): 199-210.

13. Zhao W, Zhang X, Liu J, Sun B, Tang H, et al. (2016) miR-27a-mediated antiproliferative effects of metformin on the breast cancer cell line MCF7. Oncol Rep. 36(6): 3691-3699.

14. Wahdan Alaswad RS, Cochrane DR, Spoelstra NS, Howe EN, Edgerton SM, et al. (2014) Metformin-induced killing of triple-negative breast cancer cells is mediated by reduction in fatty acid synthase via miRNA-193b. Horm Cancer 5(6): 374-389.

15. Hedrick E, Cheng Y, Jin UH, Kim K, Safe S (2016) Specificity protein (Sp) transcription factors Sp1, Sp3 and Sp4 are non-oncogene addiction genes in cancer cells. Oncotarget 7(16): 22245-22256.

16. Safe S, Nair V, Karki K (2018) Metformin-induced anticancer activities: recent insights. Biol Chem 399(4): 321-335.

17. Nair V, Pathi S, Jutooru I, Sreevalsan S, Basha R, (2013) Metformin inhibits pancreatic cancer cell and tumor growth and downregulates $\mathrm{Sp}$ transcription factors. Carcinogenesis 34(12): 2870-2879.

18. Wang J, Gao Q Wang D, Wang Z, Hu C (2015) Metformin inhibits growth of lung adenocarcinoma cells by inducing apoptosis via the mitochondriamediated pathway. Oncol Lett 10(3): 1343-1349.

19. Chai X, Chu H, Yang X, Meng Y, Shi P, et al. (2015) Metformin Increases Sensitivity of Pancreatic Cancer Cells to Gemcitabine by Reducing CD133+ Cell Populations and Suppressing ERK/P70S6K Signaling. Sci Rep 5: 14404

20. Gu Y, Zhang B, Gu G, Yang X, Qian Z (2020) Metformin Increases the Chemosensitivity of Pancreatic Cancer Cells to Gemcitabine by Reversing EMT Through Regulation DNA Methylation of miR-663. Onco Targets Ther 13: 10417-10429.

21. Saini N, Yang X (2018) Metformin as an anti-cancer agent: actions and mechanisms targeting cancer stem cells. Acta Biochim Biophys Sin (Shanghai). 50(2): 133-143.

22. Mitsuhashi A, Sato Y, Kiyokawa T, Koshizaka M, Hanaoka H, et al. (2016) Phase II study of medroxyprogesterone acetate plus metformin as a fertility-sparing treatment for atypical endometrial hyperplasia and endometrial cancer. Ann Oncol 27(2): 262-266.

23. Owen MR, Doran E, Halestrap AP (2000) Evidence that metformin exerts its anti-diabetic effects through inhibition of complex 1 of the mitochondrial respiratory chain. Biochem J 348 Pt 3(Pt 3):607-614.

24. Zhou G, Myers R, Li Y, Chen Y, Shen X, et al. (2001) Role of AMP-activated protein kinase in mechanism of metformin action. J Clin Invest 108(8): 1167-1174.

25. Markowicz Piasecka M, Sikora J, Mateusiak Ł, Mikiciuk Olasik E, Huttunen KM (2017) Metformin, and Its Sulfenamide Prodrugs Inhibit Human Cholinesterase Activity. Oxid Med Cell Longev 2017: 7303096.

26. Pollak MN (2012) Investigating metformin for cancer prevention and treatment: the end of the beginning. Cancer Discov 2(9): 778-790.
27. Nies AT, Hofmann U, Resch C, Schaeffeler E, Rius M, et al. (2011) Proton pump inhibitors inhibit metformin uptake by organic cation transporters (OCTs). PLoS One 6(7): e22163.

28. Graham GG, Punt J, Arora M, Day RO, Doogue MP, et al. (2011) Clinical pharmacokinetics of metformin. Clin Pharmacokinet 50(2): 81-98.

29. Aoki M, Terada T, Kajiwara M, Ogasawara K, Ikai I, et al. (2008) Kidneyspecific expression of human organic cation transporter 2 (OCT2/ SLC22A2) is regulated by DNA methylation. Am J Physiol Renal Physiol 295(1): F165-F170.

30. Sprowl JA, Van Doorn L, Hu S, Van Gerven L, De Bruijn P, et al. (2013) Conjunctive therapy of cisplatin with the OCT2 inhibitor cimetidine: influence on antitumor efficacy and systemic clearance. Clin Pharmacol Ther 94(5): 585-592.

31. Sleijfer DT, Offerman JJ, Mulder NH, Verweij M, Van Der Hem GK, et al. (1987) The protective potential of the combination of verapamil and cimetidine on cisplatin-induced nephrotoxicity in man. Cancer 60(11): 2823-2828.

32. Ciarimboli G, Deuster D, Knief A, Sperling M, Holtkamp M, et al. (2010) Organic cation transporter 2 mediates cisplatin-induced oto- and nephrotoxicity and is a target for protective interventions. Am J Pathol 176(3): 1169-1180.

33. Malin SK, Stewart NR (2020) Metformin May Contribute to Interindividual Variability for Glycemic Responses to Exercise. Front Endocrinol (Lausanne) 11: 519

34. Wang J, Xia S, Zhu Z (2015) Synergistic effect of phenformin in non-small cell lung cancer (NSCLC) ionizing radiation treatment. Cell Biochem Biophys 71(2): 513-518.

35. García Rubiño ME, Carrillo E, Ruiz Alcalá G, Domínguez Martín A, A Marchal J, et al. (2019) Phenformin as an Anticancer Agent: Challenges and Prospects. Int J Mol Sci 20(13): 3316

36. Duong JK, Furlong TJ, Roberts DM, Graham GG, Greenfield JR, et al. (2013) The Role of Metformin in Metformin-Associated Lactic Acidosis (MALA): Case Series and Formulation of a Model of Pathogenesis 36(9): 733-746.

37. Lam TG, Jeong YS, Kim SA, Ahn SG (2018) New metformin derivative HL156A prevents oral cancer progression by inhibiting the insulin-like growth factor/AKT/mammalian target of rapamycin pathways. Cancer Sci 109(3): 699-709.

38. Cheng G, Zielonka J, Ouari O, Lopez M, McAllister D, et al. (2016) Mitochondria-Targeted Analogues of Metformin Exhibit Enhanced Antiproliferative and Radiosensitizing Effects in Pancreatic Cancer Cells. Cancer Res 76(13): 3904-3915.

39. Zhang L, Han J, Jackson AL, Clark LN, Kilgore J, et al. (2016) NT1014, a novel biguanide, inhibits ovarian cancer growth in vitro and in vivo. J Hematol Oncol 9(1): 91.

40. Zhang L, Han J, Jackson AL, Clark LN, Kilgore J, et al. (2016) NT1014, a novel biguanide, inhibits ovarian cancer growth in vitro and in vivo. J Hematol Oncol 9(1): 91. 